\title{
Charged lepton flavor violation in a class of radiative neutrino mass generation models
}

\author{
Talal Ahmed Chowdhury ${ }^{1,2, *}$ and Salah Nasri ${ }^{3,2, \dagger}$ \\ ${ }^{1}$ Department of Physics, University of Dhaka, P.O. Box 1000 Dhaka, Bangladesh \\ ${ }^{2}$ Abdus Salam International Centre for Theoretical Physics, Strada Costiera 11, I-34014 Trieste, Italy \\ ${ }^{3}$ Department of Physics, UAE University, P.O. Box 17551 Al-Ain, United Arab Emirates
}

(Received 29 January 2018; published 30 April 2018)

\begin{abstract}
We investigate the charged lepton flavor violating processes $\mu \rightarrow e \gamma, \mu \rightarrow e e \bar{e}$, and $\mu-e$ conversion in nuclei for a class of three-loop radiative neutrino mass generation models with electroweak multiplets of increasing order. We find that, because of certain cancellations among various one-loop diagrams which give the dipole and nondipole contributions in an effective $\mu e \gamma$ vertex and a Z-penguin contribution in an effective $\mu e Z$ vertex, the flavor violating processes $\mu \rightarrow e \gamma$ and $\mu-e$ conversion in nuclei become highly suppressed compared to $\mu \rightarrow e e \bar{e}$ process. Therefore, the observation of such a pattern in LFV processes may reveal the radiative mechanism behind neutrino mass generation.
\end{abstract}

DOI: 10.1103/PhysRevD.97.075042

\section{INTRODUCTION}

Although we have observed lepton flavor violation (LFV) in the neutral fermion sector of the Standard Model (SM) in neutrino oscillation, the charged LFV in the SM has turned out to be highly suppressed. For example, by allowing massive neutrinos, $m_{\nu} \sim 1 \mathrm{eV}$ and the leptonic mixing matrix, Pontecorvo-Maki-NakagawaSakata (PMNS) matrix, $U_{\text {PMNS }}$ in the SM, the branching ratio of the charged lepton violating process, $\mu \rightarrow e \gamma$, turns out to be about $10^{-47}$ [1-5], which is beyond any experimental reach in the foreseeable future. But many physics beyond the standard model (BSM) scenario, especially new physics related to the generation and smallness of the neutrino mass, can lead to unsuppressed charged LFV processes $[2,6,7] .{ }^{1}$ which are within the reach of currently operating and future experiments. For theoretical and experimental status of charged LFV, please see [9-14].

A well motivated model of neutrino mass generation which addresses the origin of the neutrino mass and the particle nature of the dark matter (DM) in our Universe, is the Krauss-Nasri-Trodden (KNT) model [15], where DM

\footnotetext{
*talal@du.ac.bd

† snasri@uaeu.ac.ae

${ }^{1}$ For a general condition of tree-level and one-loop lepton flavor violating processes, please see [8].

Published by the American Physical Society under the terms of the Creative Commons Attribution 4.0 International license. Further distribution of this work must maintain attribution to the author(s) and the published article's title, journal citation, and DOI. Funded by SCOAP ${ }^{3}$.
}

particles radiatively generate the mass of the neutrino at three loops and additional BSM particles having mass at the $\mathrm{O}(\mathrm{TeV})$ range, can be accessible to the $\mathrm{LHC}$ or future hadron colliders ${ }^{2}$ In [15], the additional BSM fields are two charged singlets $S_{1}^{+}$and $S_{2}^{+}$and three fermion singlets $N_{R_{1,2,3}}$ which are right-handed (rh) neutrinos. A $Z_{2}$ symmetry with an action $\left\{S_{2}^{+}, N_{R_{i}}\right\} \rightarrow\left\{-S_{2}^{+},-N_{R_{i}}\right\}$ is also imposed to prevent the tree-level Dirac mass of the neutrino after electroweak symmetry breaking and ensures stability of the lightest rh neutrino, $N_{R_{1}}$, thereby giving a DM candidate.

Consequently, the three-loop topology of a radiative neutrino mass diagram remains invariant [17], if one replaces $S_{2}^{+}$with a larger scalar multiplet, $\boldsymbol{\Phi}$, which has an integer isospin, $j_{\phi}$ and hypercharge, ${ }^{3} Y_{\phi}=1$ under $S U(2)_{L} \times U(1)_{Y}$, and $N_{R_{i}}$ are replaced with a fermionic multiplet $\mathbf{F}_{\mathbf{i}}, i=1,2,3$, with an integer isospin, $j_{F}$ and hypercharge, $Y_{F}=0$. In this scenario, the DM candidate is the lightest neutral component of $\mathbf{F}_{1}$, i.e., $F_{1}^{0}$. Therefore, the immediate generalization of the KNT model is [18], where the particle content is taken as, $\boldsymbol{\Phi}$ with $\left(j_{\phi}, Y_{\phi}\right)=(1,1)$ and $\mathbf{F}_{\mathbf{i}}$ with $\left(j_{F}, Y_{F}\right)=(1,0)$. Here, $Z_{2}$ symmetry is still needed to enforce the Dirac mass term of neutrinos to be zero at tree level.

In addition, no Yukawa terms with a SM fermion that give rise to the Dirac neutrino mass, are allowed in the Lagrangian if the KNT particle content is extended with $\boldsymbol{\Phi}$ that has $\left(j_{\phi}, Y_{\phi}\right)=(2,1)$ and $\mathbf{F}_{\mathbf{i}}$ with $\left(j_{F}, Y_{F}\right)=(2,0)$ to

\footnotetext{
${ }^{2}$ For a recent review on radiative generation of neutrino mass, please see [16].

${ }^{3}$ Here, the electric charge is $Q=T^{3}+Y$.
} 
generate the neutrino mass at a three-loop level [19]. Therefore, there is no need to use $Z_{2}$ symmetry for that purpose. But the viable dark matter candidate in the model, which is a $F_{1}^{0}$ Majorana fermion, has a one-loop decay process which depends on the $\lambda S_{1}^{-} \boldsymbol{\Phi}^{\dagger}$. $\boldsymbol{\Phi} . \boldsymbol{\Phi}$ term in the scalar potential. From the bound on the dark matter mean lifetime [20], which is of the order $10^{25}-10^{27} \mathrm{sec}$, the $\lambda$ coupling has to be $\lambda \sim 10^{-26}-10^{-27}$ for $\mathrm{TeV}$ mass-ranged DM. Moreover, the neutrino sector of the model does not depend on this coupling in any way. Therefore in the limit $\lambda \rightarrow 0$, the softly broken accidental $Z_{2}$ symmetry becomes exact and ensures the stability of the DM.

Consequently, one can go to the next higher scalar and fermion representations in this class of generalized KNT models. In the case of $\boldsymbol{\Phi}$ with $\left(j_{\phi}, Y_{\phi}\right)=(3,1)$ and $\mathbf{F}_{\mathbf{i}}$ with $\left(j_{F}, Y_{F}\right)=(3,0)$, the field content of the model not only prevents the appearance of Dirac mass term for a neutrino but also the $\lambda$ term in the scalar potential which would have prevented DM to be absolutely stable [21]. The direct product of two $S U(2)$ scalar representations, $\boldsymbol{\Phi} \otimes \boldsymbol{\Phi}$ gives $j_{\phi} \otimes j_{\phi}=\oplus_{J} J \supset j_{\phi}^{\prime}$, where $j_{\phi}^{\prime}$ has the same isospin value as $j_{\phi}$ and therefore forms an invariant in the term $\lambda S_{1}^{-} \boldsymbol{\Phi}^{\dagger} \otimes \boldsymbol{\Phi} \otimes \boldsymbol{\Phi}$ but it is either symmetric or antisymmetric representation depending on the even-integer or odd-integer isospin value $j_{\phi}$, respectively. As the antisymmetrized $\boldsymbol{\Phi} \otimes \boldsymbol{\Phi}$ representations are identically zero for an odd-integer isospin, the $\lambda$ term does not appear in the scalar potential at a renormalizable level and the DM is stable.

The main motivation of this paper is to carry out a comparative study of charged lepton flavor violating processes in this class of generalized KNT models with singlet, triplet, 5 plet, and 7 plet. The most studied charged LFV processes are $\mu \rightarrow e \gamma, \mu \rightarrow e e \bar{e}$, and $\mu-e$ conversion in the nuclei. The MEG Collaboration has put a bound on the $\mu \rightarrow e \gamma$ process as $\operatorname{Br}(\mu \rightarrow e \gamma)<4.2 \times 10^{-13}$ at 90\% C.L. [22]. In addition, the process $\mu \rightarrow e e \bar{e}$ has a current limit as, $\operatorname{Br}(\mu \rightarrow e e \bar{e})<1.0 \times 10^{-12}(90 \%$ C.L.) set by the SINDRUM Collaboration [23]. Moreover, $\mu-e$ conversion processes in nuclei, $\mu \mathrm{Au}, \mathrm{Ti} \rightarrow e \mathrm{Au}, \mathrm{Ti}$ have limits on rates, $\mathrm{CR}(\mu-e, \mathrm{Au})<7 \times 10^{-13}$ (90\% C.L.) [24] and $\mathrm{CR}(\mu-e, \mathrm{Ti})<6.1 \times 10^{-13}$ (90\% C.L.) [25] set by the SINDRUM II Collaboration. On the other hand, the future reach on $\mu \rightarrow e \gamma$ is, $\operatorname{Br}(\mu \rightarrow e \gamma)<5.4 \times 10^{-14}$ by the MEG II experiment, which will start taking data from 2018 [26]. The Mu3e experiment, which will begin its run on 2019, will have a reach $\operatorname{Br}(\mu \rightarrow e e \bar{e})<10^{-16}$ [27]. In addition, the $\mu-e$ conversion experiment Mu2e, which is scheduled to start on 2020, will have $\mathrm{CR}(\mu-e, \mathrm{Al})<$ $6.7 \times 10^{-17}$ [28]. For this reason, we have systematically studied these three processes in each case of a generalized KNT model with respect to the current bounds and future sensitivity limits.

In this article, we present the generalized KNT model with larger electroweak multiplets in Sec. II. In Sec. III, we describe the relevant formulas of charged LFV processes $\mu \rightarrow e \gamma, \mu \rightarrow e e \bar{e}$, and $\mu-e$ conversion rate in nuclei in a generalized KNT model. Section IV contains the result of charged LFV processes in this model. We conclude in Sec. V. Appendix contains the loop functions used in calculations of charged LFV processes.

\section{THE MODEL}

\section{A. The field content}

The three-loop radiative neutrino mass generation model contains a charged singlet $S_{1}^{+} \sim(0,0,1)$, a complex scalar multiplet, $\boldsymbol{\Phi} \sim\left(0, j_{\phi}, 1\right)$, and three real fermion multiplets, $\mathbf{F}_{1,2,3} \sim\left(0, j_{F}, 0\right)$ under $S U(3)_{c} \times S U(2)_{L} \times U(1)_{Y}$. The multiplets are

$$
\begin{aligned}
\boldsymbol{\Phi} & =\left(\phi^{\left(j_{\phi}+1\right)}, \phi^{\left(j_{\phi}\right)}, \ldots, \phi^{0}, \ldots, \phi^{\left(-j_{\phi}+2\right)}, \phi^{\left(-j_{\phi}+1\right)}\right)^{T} \\
\mathbf{F}_{1,2,3} & =\left(F^{\left(j_{F}\right)}, F^{\left(j_{F}-1\right)}, \ldots, F^{0}, \ldots, F^{\left(-j_{F}+1\right)}, F^{\left(-j_{F}\right)}\right)_{1,2,3}^{T} .
\end{aligned}
$$

In this comparative study, we focus on four set of models in this class which we have referred as

\begin{tabular}{lcc}
\hline Model & $\mathbf{\Phi}$ & $\mathbf{F}_{1,2,3}$ \\
\hline Singlet & $(0,0,1)$ & $(0,0,0)$ \\
Triplet & $(0,1,1)$ & $(0,1,0)$ \\
5 plet & $(0,2,1)$ & $(0,2,0)$ \\
7 plet & $(0,3,1)$ & $(0,3,0)$ \\
\hline
\end{tabular}

The SM Lagrangian is extended in the following way:

$$
\begin{aligned}
\mathcal{L} \supset & \mathcal{L}_{\mathrm{SM}}+\left\{f_{\alpha \beta} \overline{L_{\alpha}^{c}} \cdot L_{\beta} S_{1}^{+}+g_{i \alpha} \overline{\mathbf{F}_{i}} \cdot \boldsymbol{\Phi} . e_{\alpha_{R}}+\text { H.c. }\right\} \\
& -\frac{1}{2} \overline{\mathbf{F}_{i}^{c}} M_{F_{i j}} \mathbf{F}_{j}-V\left(H, \boldsymbol{\Phi}, S_{1}\right)+\text { H.c. }
\end{aligned}
$$

where, $\mathrm{c}$ denotes the charge conjugation and the dot sign, in shorthand, refers to appropriate $S U(2)$ contractions. Also $L_{\alpha}$ and $e_{R_{\alpha}}$ are the lh lepton doublet and rh charged leptons, respectively, and the Greek alphabet $\alpha$ stands for the generation index. Moreover, $[F]_{\alpha \beta}=f_{\alpha \beta}$ and $[G]_{i \alpha}=g_{i \alpha}$ are $3 \times 3$ complex antisymmetric and general complex matrices, respectively. Finally, $H$ denotes the SM Higgs doublet.

The scalar potential is given by

$$
\begin{aligned}
V\left(H, \boldsymbol{\Phi}, S_{1}\right)= & V(H)+V(\boldsymbol{\Phi})+V\left(S_{1}\right)+V_{1}(H, \boldsymbol{\Phi}) \\
& +V_{2}\left(H, S_{1}\right)+V_{3}\left(\boldsymbol{\Phi}, S_{1}\right) .
\end{aligned}
$$

The three-loop neutrino mass generation depends on the $V_{3}$ term as follows:

$$
V_{3} \supset \frac{\lambda_{S}}{4}\left(S_{1}^{-}\right)^{2} \boldsymbol{\Phi} . \boldsymbol{\Phi}+\text { H.c. }
$$




\section{B. Mass splittings in the multiplets}

At the tree level, the components of the fermion multiplet, $\mathbf{F}_{i}$ are mass degenerate. Moreover, we work in the generation basis, where $M_{F_{i j}}=\operatorname{diag}\left(M_{F_{1}}, M_{F_{2}}, M_{F_{3}}\right)$. We have also considered the nondegenerate mass for the three fermion multiplets, $M_{F_{1}}<M_{F_{2}}<M_{F_{3}}$.

Consequently, after electroweak symmetry breaking (EWSB), the radiative corrections, for example, loops involving SM gauge bosons, lift the mass degeneracy in the component fields of the fermion multiplets. In the limit, $M_{F} \gg M_{W}$, the mass splitting between the components of charge $Q$ and $Q^{\prime}$ is, $M_{Q}-M_{Q^{\prime}} \sim\left(Q^{2}-Q^{\prime 2}\right) \Delta$, where, $\Delta \equiv \alpha_{W} \sin ^{2}\left(\theta_{w} / 2\right) M_{W} \sim 166 \mathrm{MeV}$ [29].

On the other hand, the component fields of the scalar multiplet, after EWSB, can have splittings at the tree level due to the following term in $V_{1}(H, \boldsymbol{\Phi})$ :

$$
V_{1}(H, \boldsymbol{\Phi}) \supset \lambda_{H \phi_{2}}\left(\boldsymbol{\Phi}^{*} . H\right) .\left(H^{*} . \boldsymbol{\Phi}\right) .
$$

The maximum splitting among the masses of the component fields in the electroweak multiplet is bounded by the constraints on the electroweak precision observables (EWPO) [30,31]. Here we consider the constraint on the $\mathrm{T}$ parameter as it is the most sensitive EWPO on mass splitting in a scalar multiplet or in other words, isospin breaking in the multiplet. From Fig. 1, we see that the larger value of the coupling, $\lambda_{H \phi 2}$, leads to the larger splitting in the scalar component fields in the multiplet. On the other hand, if $M_{0}=10 \mathrm{TeV}$ and $\lambda_{H \phi 2}=2 \pi$, the splitting between any two components of the scalar multiplet, allowed by the EWPO constraints, is very small as $\Delta m_{i j}^{2} / M_{0}^{2} \sim 10^{-3}$. Again, with $M_{F_{i}} \sim 10 \mathrm{TeV}$, the radiative mass splittings between two components of the fermionic multiplet leads to $\Delta m_{F_{i j}}^{2} / M_{0}^{2} \sim 10^{-4}$. Therefore, for the scalar and fermion multiplets' mass in the $\mathrm{TeV}$ range, the mass splittings are numerically negligible; therefore, we consider this scenario as the "near degenerate" case and make proper approximations in our subsequent analysis.

\section{Three-loop radiative neutrino mass}

The neutrino mass is generated radiatively at three loops. In the near degenerate case, we neglect the small mass splittings and have $[15,18,19,21]$

$\left(M_{\nu}\right)_{\alpha \beta}=\frac{c \lambda_{S}}{\left(4 \pi^{2}\right)^{3}} \frac{m_{\gamma} m_{\delta}}{M_{\phi}} f_{\alpha \gamma} f_{\beta \delta} g_{\gamma i}^{*} g_{\delta i}^{*} F\left(\frac{M_{F_{i}}^{2}}{M_{\phi}^{2}}, \frac{m_{S}^{2}}{M_{\phi}^{2}}\right)$,

where, $c=1, c=3, c=5$, and $c=7$ are for singlet, triplet, 5-plet, and 7-plet cases, respectively. Equation (6) can be written in compact form,

$$
M_{\nu}=X . \Lambda . X^{T}, \quad \text { with } \quad X=F M_{l} G^{\dagger} .
$$

Here, $M_{l}$ is the diagonal charged lepton mass matrix and $\Lambda=\operatorname{diag}\left(\Lambda_{1}, \Lambda_{2}, \Lambda_{3}\right)$, where $\Lambda_{i}$ is associated with $\mathbf{F}_{i}$.

The loop function $F$ with $\alpha=M_{F_{i}}^{2} / M_{\phi}^{2}$ and $\beta=m_{S}^{2} / M_{\phi}^{2}$ is given by

$$
F(\alpha, \beta)=\frac{\sqrt{\alpha}}{8 \beta^{2}} \int_{0}^{\infty} d r \frac{r}{r+\alpha} I(r, \beta)^{2},
$$

and the function $I(r, \beta)$ is

$$
\begin{aligned}
I(r, \beta)= & \ln \left[r\left(\eta_{+}-1\right)\left(1-\eta_{-}\right)\right]-\eta_{+} \ln \left[\frac{\eta_{+}-1}{\eta_{+}}\right] \\
& -\eta_{-} \ln \left[\frac{\eta_{-}-1}{\eta_{-}}\right]-\frac{1+r}{r} \ln [1+r],
\end{aligned}
$$

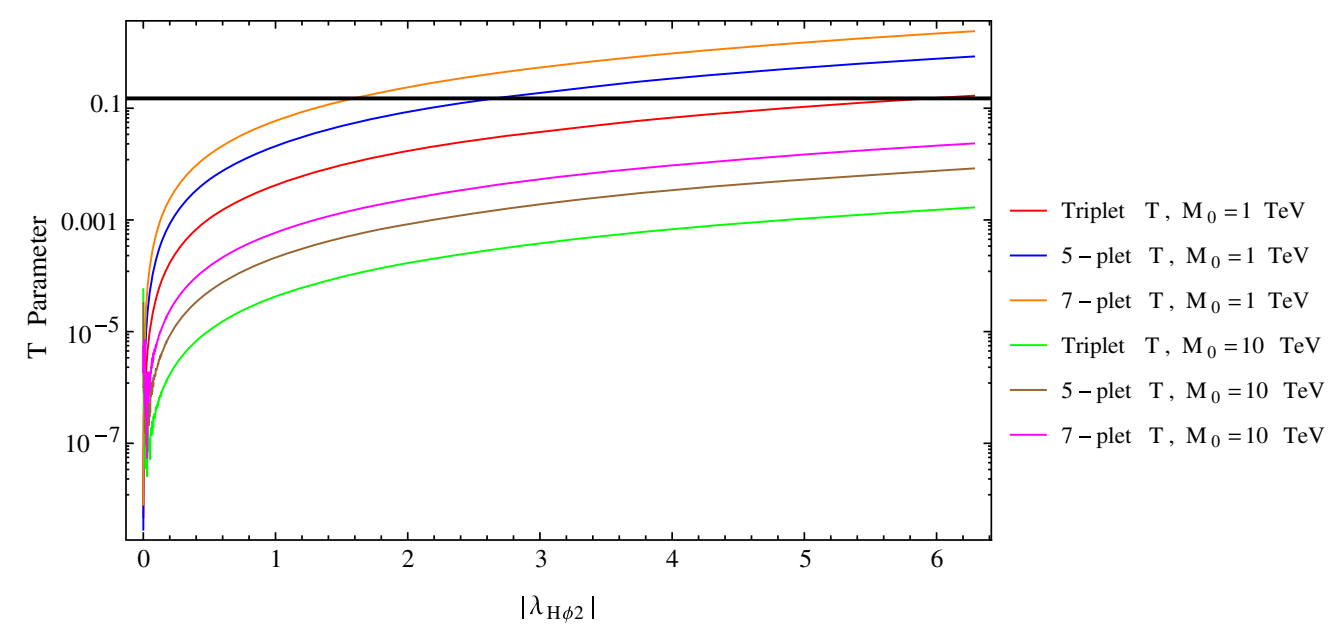

FIG. 1. Correlation between $\lambda_{H \phi 2}$ and $T$ parameter. Here, we have used two values of $M_{0}$ which is the invariant mass from the Lagrangian, $M_{0}=1 \mathrm{TeV}$ and $M_{0}=10 \mathrm{TeV}$, respectively. Also the black line represents the maximum bound on the $\mathrm{T}$ parameter, $T=0.07 \pm 0.08$ (68\% C.L.) [32]. 


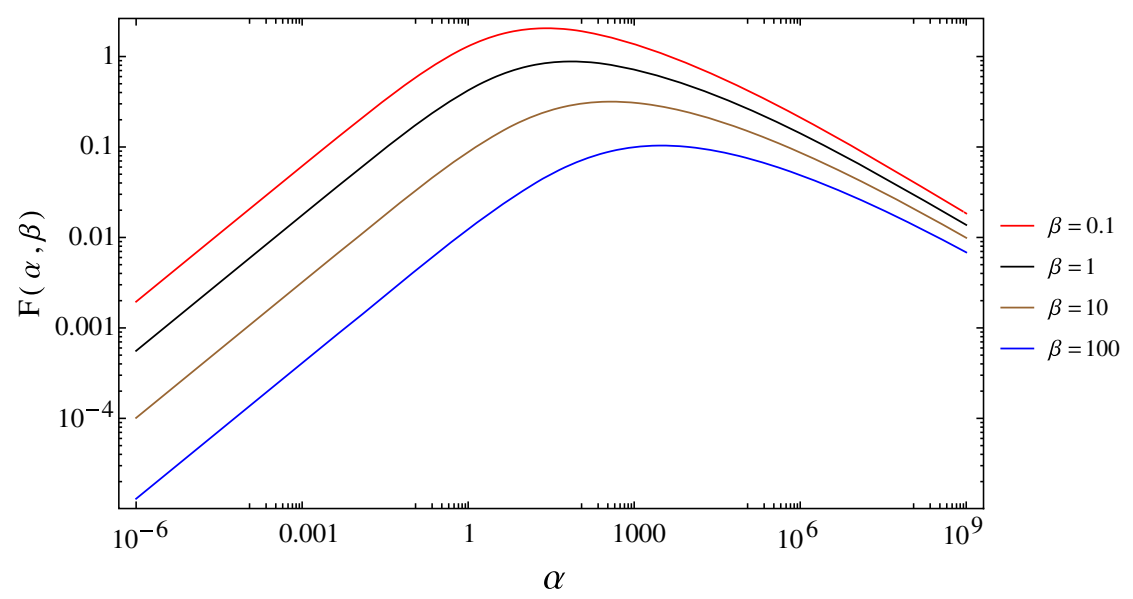

FIG. 2. The function $F(\alpha, \beta)$.

where

$\eta_{ \pm}(r, \beta)=\frac{1}{2 r}\left(1+r-\beta \pm \sqrt{(1+r-\beta)^{2}+4 r \beta}\right)$.

The behavior of function $F(\alpha, \beta)$ with $\alpha$ and $\beta$ is shown in Fig. 2.

The neutrino mass matrix, $M_{\nu}$ of Eq. (6), can be diagonalized as

$$
M_{\nu}=U_{\mathrm{PMNS}} \cdot \hat{m}_{\nu} \cdot U_{\mathrm{PMNS}}^{T},
$$

where $\hat{m}_{\nu}=\operatorname{diag}\left(m_{\nu_{1}}, m_{\nu_{2}}, m_{\nu_{3}}\right)$, and it contains seven independent parameters which are two masses $m_{\nu_{2,3}}$ that can be determined assuming either normal or inverted hierarchy by using experimentally measured [32] two mass squared differences $\Delta m_{\text {atm }}^{2}$ and $\Delta m_{\text {solar }}^{2}$, three mixing angles $\theta_{12}, \theta_{23}$, and $\theta_{13}$ and still to be determined, one Dirac phase $\delta_{\mathrm{CP}}$, and one Majorana phase $\alpha_{M}$ in $U_{\mathrm{PMNS}}$ matrix. Here, due to $\operatorname{det} F=0$, the lowest neutrino mass is $m_{\nu_{1}}=0$, and it also implies one Majorana phase of $U_{\text {PMNS }}$ to be zero. On the other hand, the matrices $F$ contains six and $G$ contains 18 degrees of freedom. As there is no one-to-one correspondence between low energy neutrino parameters in $M_{\nu}$ and the parameters of $F, G$, and $\Lambda$, we numerically determine the set $\left\{f_{\alpha \beta}, g_{i \alpha}, M_{F_{1,2,3}}, M_{\phi}, m_{S}, \lambda_{S}\right\}$, which satisfy the following relation:

$$
\operatorname{Tr}\left(M_{\nu}^{\dagger} M_{\nu}\right)=\operatorname{Tr}\left(X^{*} \Lambda X^{\dagger} X \Lambda X^{T}\right) .
$$

We have used this relation because there are no unique $F$ and $G$ which satisfy the low energy neutrino constraints, $U_{\text {PMNS }}$. Therefore, one can always find another set of $F^{\prime}$ and $G^{\prime}$ through orthogonal transformation, $F^{\prime} \rightarrow V F V^{T}$ and biunitary transformation, $G^{\prime} \rightarrow W G Y^{\dagger}$ where, $V$, $W$, and $Y$ are unitary matrices, to satisfy the low energy constraints.
As seen from Eq. (6), the generalized KNT model with a larger multiplet does not qualitatively change the structure of the neutrino mass matrix. For each multiplet, the numerical change of the order unity in $M_{\nu}$ comes from slight shifts in $F, G$ Yukawa couplings and the mass ratios entering in the function $F$ of Eq. (8) while satisfying the low energy neutrino constraints. However, in the particular case of the 5-plet and 7-plet representations, there are no Yukawa term with SM leptons invariant under the SM gauge group, which can lead to a Dirac neutrino mass at tree level after electroweak symmetry breaking and that would have spoiled the radiative neutrino mass generation setup. Therefore, the ad hoc $Z_{2}$ symmetry which is assumed to prevent the Dirac mass term in the singlet case is not required for the generalized KNT model with 5-plet and 7-plet matter fields.

\section{CHARGED LEPTON FLAVOR VIOLATING PROCESSES}

As the charged LFV processes, $\mu \rightarrow e \gamma, \mu \rightarrow e e \bar{e}$, and $\mu-e$ conversion in $\mathrm{Au}$ and $\mathrm{Ti}$ nuclei, have the most stringent experimental constraints, we focus our study on these three LFV processes in the generalized KNT model with a singlet, triplet, 5 plet, and 7 plet, respectively.

$$
\text { A. } \mu \rightarrow \boldsymbol{e} \gamma
$$

The branching ratio for $\mu \rightarrow e \gamma$, normalized by $\operatorname{Br}\left(\mu \rightarrow e \overline{\nu_{e}} \nu_{\mu}\right)$, is

$$
\operatorname{Br}(\mu \rightarrow e \gamma)=\frac{3(4 \pi)^{3} \alpha_{e m}}{4 G_{F}^{2}}\left|A_{D}\right|^{2} \operatorname{Br}\left(\mu \rightarrow e \nu_{\mu} \overline{\nu_{e}}\right),
$$

where

$$
A_{D}=A_{D}^{(1)}+A_{D}^{(2)}+A_{D}^{(3)}
$$

where 


$$
\begin{aligned}
A_{D}^{(1)} & =\sum_{i=1}^{3} \sum_{\phi} \frac{g_{e i}^{*} g_{i \mu} q_{\phi}}{32 \pi^{2}} \frac{1}{m_{\phi}^{2}} F_{1}\left(x_{i \phi}^{q}\right) \\
A_{D}^{(2)} & =-\sum_{i=1}^{3} \sum_{F_{i}} \frac{g_{i i}^{*} g_{i \mu} q_{F_{i}}}{32 \pi^{2}} \frac{1}{m_{\phi}^{2}} F_{2}\left(x_{i \phi}^{q}\right) \\
A_{D}^{(3)} & =\frac{f_{e \tau}^{*} f_{\tau \mu}}{192 \pi^{2}} \frac{1}{m_{S}^{2}}
\end{aligned}
$$

where $m_{\phi}$ and $q_{\phi}\left(q_{F}\right)$ are the corresponding mass and the electric charge, respectively, of the scalar (fermion) component $\phi^{(q)}\left(F_{i}^{(q)}\right), x_{i \phi}^{q}=m_{F_{i}^{(q-1)}}^{2} / m_{\phi^{(-q)}}^{2}$, and $\phi\left(F_{i}\right)$ runs over all the charged components of the scalar (fermion) multiplet $\boldsymbol{\Phi}\left(\mathbf{F}_{i}\right)$. Note that $A_{D}^{(1)}$ and $A_{D}^{(2)}$ involve rh charged leptons, whereas, $A_{D}^{(3)}$ involves lh charged leptons.

Figures 3(I) and 3(II), where an external $\gamma$ line is attached to charged scalars and charged fermions, respectively, and give dipole contributions $A_{D}^{(1)}$ and $A_{D}^{(2)}$ that come from pairs, $\left[\phi^{(-q)}, F_{i}^{(q-1)}\right]$, where, $q=-j_{\phi}+1, \ldots, j_{\phi}+1$. Since the mass splittings among the component fields of both the scalar and fermion multiplet are small as pointed out in Sec. II B, we can consider the near degenerate limit, and in this case, there are cancellations in $A_{D}^{(2)}$ because degenerate fermion components of opposite electric charge have a photon line attached to it and therefore, the sum over all fermion components renders it to $A^{(2)} \sim 0$. Moreover, the same cancellations take place in $A_{D}^{(1)}$ when scalar components of an opposite electric charge have a photon line attached to it. Therefore, in the case of a triplet, we have non-negligible contributions from $\left(\phi^{--}, F_{i}^{+}\right)$and $\left(\phi^{-}, F_{i}^{0}\right)$ pairs in $A_{D}^{(1)}$. For the 5 plet, we have nonnegligible contributions in $A_{D}^{(1)}$ from $\left(\phi^{---}, F_{i}^{++}\right)$and $\left(\phi^{--}, F_{i}^{+}\right)$pairs. Finally, for the 7 plet, the only nonnegligible contributions in $A_{D}^{(1)}$ come from $\left(\phi^{----}, F_{i}^{+++}\right)$ and $\left(\phi^{---}, F_{i}^{++}\right)$pairs. On the other hand, the singlet case only involves a $\left(\phi^{-}, F_{i}^{0}\right)$ pair as the nonzero contribution to $A_{D}^{(1)}$. On the other hand, Fig. 3(III) gives the dipole contribution $A_{D}^{(3)}$ coming from $\left(S_{1}^{-}, \nu_{\tau}\right)$ pair for all cases.

\section{B. $\mu \rightarrow e e \bar{e}$}

In the generalized KNT model, the three-body lepton flavor violating decay mode $\mu \rightarrow e e \bar{e}$ receives the contributions from $\gamma$-penguin diagrams, Z-penguin diagrams, and box diagrams. In this model, a Higgs penguin diagram does not contribute to this process. Therefore, the branching ratio of $\mu \rightarrow e e \bar{e}$ is given [33-36] as

$$
\begin{aligned}
\operatorname{Br}(\mu \rightarrow e e \bar{e}) \\
=\frac{3(4 \pi)^{2} \alpha_{e m}^{2}}{8 G_{F}^{2}}\left[\left|A_{N D}\right|^{2}+\left|A_{D}\right|^{2}\left(\frac{16}{3} \ln \frac{m_{\mu}}{m_{e}}-\frac{22}{3}\right)\right. \\
\quad+\frac{1}{6}|B|^{2}+\frac{1}{3}\left(2\left|F_{Z}^{L}\right|^{2}+\left|F_{Z}^{R}\right|^{2}\right) \\
\left.\quad+\left(-2 A_{N D} A_{D}^{*}+\frac{1}{3} A_{N D} B^{*}-\frac{2}{3} A_{D} B^{*}+\text { H.c. }\right)\right] \\
\quad \times \operatorname{Br}\left(\mu \rightarrow e \bar{\nu}_{e} \nu_{\mu}\right),
\end{aligned}
$$

where $A_{D}$ and $A_{N D}$ are the dipole and nondipole contributions from the photonic penguin diagrams, respectively. $F_{Z}^{L}$ and $F_{Z}^{R}$ are given as

$$
F_{Z}^{L}=\frac{F_{Z} g_{L}^{l}}{g^{2} m_{Z}^{2} \sin ^{2} \theta_{W}}, \quad F_{Z}^{R}=\frac{F_{Z} g_{R}^{l}}{g^{2} m_{Z}^{2} \sin ^{2} \theta_{W}} .
$$

Here, $F_{Z}$ is the Z-penguin contribution and $g_{L}^{l}$ and $g_{R}^{l}$ are the Z-boson coupling to the left-handed (lh) and righthanded (rh) charged leptons, respectively. Also $B$ represents the contribution from the box diagrams.

\section{1. $\gamma$-penguin contribution}

The $\gamma$ penguin diagram can be obtained by attaching the $e-\bar{e}$ fermion line to $\gamma$ line in Figs. 3(I), 3(II), and 3(III). The dipole contribution of $\gamma$-penguin diagrams are same as in Sec. III A. So we consider here the nondipole contribution, which is

$$
A_{N D}=A_{N D}^{(1)}+A_{N D}^{(2)}+A_{N D}^{(3)}
$$

Here,
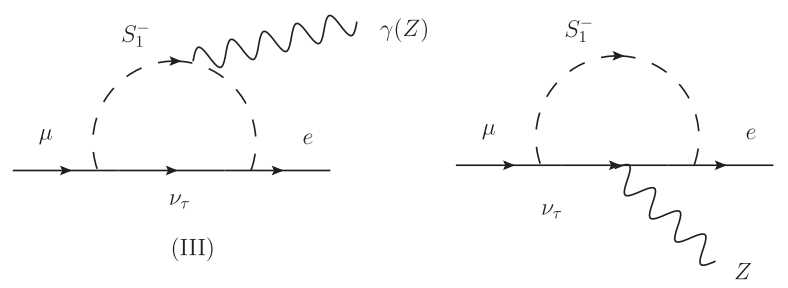

(IV)

FIG. 3. One loop diagrams contributing to effective $\mu e \gamma$ and $\mu e Z$ vertices. For simplicity, we have not included self-energy diagrams, where the $\gamma(Z)$ line is attached to external fermions. 


$$
\begin{aligned}
& A_{N D}^{(1)}=\sum_{i=1}^{3} \sum_{\phi} \frac{g_{e i}^{*} g_{i \mu} q_{\phi}}{32 \pi^{2}} \frac{1}{m_{\phi}^{2}} G_{1}\left(x_{i \phi}^{q}\right) \\
& A_{N D}^{(2)}=-\sum_{i=1}^{3} \sum_{F_{i}} \frac{g_{e i}^{*} g_{i \mu} q_{F_{i}}}{32 \pi^{2}} \frac{1}{m_{\phi}^{2}} G_{2}\left(x_{i \phi}^{q}\right) \\
& A_{N D}^{(3)}=\frac{f_{e \tau}^{*} f_{\tau \mu}}{288 \pi^{2}} \frac{1}{m_{S}^{2}} .
\end{aligned}
$$

The nondipole contributions $A_{N D}^{(1)}, A_{N D}^{(2)}$, and $A_{N D}^{(3)}$ are associated with Figs. 3(I), 3(II), and 3(III), respectively, with the $\gamma$ line having an $e-\bar{e}$ fermion line attached to it. The loop functions $G_{1}(x)$ and $G_{2}(x)$ are given in the Appendix. As in the case of dipole contributions, in the near degenerate mass limit, we again have cancellations among the charged fermions with an opposite electric charge in $A_{N D}^{(2)}$; therefore, $A_{N D}^{(2)} \sim 0$. In addition, the contributions from the charged scalar with an opposite electric charge get canceled in $A_{N D}^{(1)}$. Once more the non-negligible contributions in $A_{N D}^{(1)}$ come from $\left(\phi^{--}, F_{i}^{+}\right)$and $\left(\phi^{-}, F_{i}^{0}\right)$ in the case of triplet, $\left(\phi^{---}, F_{i}^{++}\right)$and $\left(\phi^{--}, F_{i}^{+}\right)$pairs for the case of the 5 plet, and $\left(\phi^{----}, F_{i}^{+++}\right)$and $\left(\phi^{---}, F_{i}^{++}\right)$ pairs for the case of the 7 plet, respectively.

\section{Z-penguin contribution}

The Z-penguin diagram can be obtained f.rom Figs. 3(I)3(IV) by attaching an $e-\bar{e}$ fermion line attaching to a $\mathrm{Z}$ boson line. Its contribution to $\mu \rightarrow e e \bar{e}$ can be arranged into two parts,

$$
F_{Z}=F_{Z}^{(1)}+F_{Z}^{(2)}
$$

where $F_{Z}^{(1)}$ is the contribution associated with Figs. 3(I) and 3 (II) with the $\mathrm{Z}$ line. On the other hand, $F_{Z}^{(2)}$ is the contribution associated with Figs. 3(III) (with the $\mathrm{Z}$ line) and 3(IV). They are given as,

$$
\begin{aligned}
F_{Z}^{(1)}= & -\frac{1}{16 \pi^{2}} \sum_{i=1}^{3} \sum_{\left(\phi, F_{i}\right)}\left\{g_{e i}^{*} g_{i \mu} g_{Z F_{i} \bar{F}_{i}}\left[\left(2 C_{24}\left(m_{\phi}, m_{F_{i}}, m_{F_{i}}\right)+\frac{1}{2}\right)+m_{F_{i}}^{2} C_{0}\left(m_{\phi}, m_{F_{i}}, m_{F_{i}}\right)\right]\right. \\
& \left.+2 g_{e i}^{*} g_{i \mu} g_{Z \phi} C_{24}\left(m_{F_{i}}, m_{\phi}, m_{\phi}\right)+g_{e i}^{*} g_{i \mu} g_{R}^{l} B_{1}\left(m_{F_{i}}, m_{\phi}\right)\right\}
\end{aligned}
$$

$$
\begin{aligned}
F_{Z}^{(2)}= & -\frac{1}{16 \pi^{2}} f_{e \tau}^{*} f_{\tau \mu}\left\{g_{Z \nu \bar{\nu}}\left(2 C_{24}\left(m_{S_{1}}, 0,0\right)+\frac{1}{2}\right)\right. \\
& \left.+2 g_{Z S_{1}} C_{24}\left(0, m_{S_{1}}, m_{S_{1}}\right)+g_{L}^{l} B_{1}\left(0, m_{S_{1}}\right)\right\},
\end{aligned}
$$

where the sum over pairs $\left(\phi, F_{i}\right)$ implies the pairs of component fields from the fermion and scalar multiplet entering into the one-loop process. $g Z F_{i} \bar{F}_{i}, g_{Z \phi}, g_{Z \nu \bar{\nu}}$, and $g_{Z S_{1}}$ are the $\mathrm{Z}$ coupling of charged fermion components of $\mathbf{F}_{\mathbf{i}}$, scalar components of $\boldsymbol{\Phi}$, tau neutrino, and charged scalar $S_{1}$, respectively. Moreover, $g_{L}^{l}$ and $g_{R}^{l}$ are the $\mathrm{Z}$ coupling of the left-handed and right-handed charged leptons, respectively.

In the near degenerate limit, for the triplet, the contributions from the following pairs in Eq. (25) are

$$
F_{Z}^{(1)}\left(\phi^{--}, F_{i}^{+}\right)=-F_{Z}^{(1)}\left(\phi^{0}, F_{i}^{-}\right) .
$$

For the 5 plet, the contribution in Eq. (25) from the following pairs are

$$
\begin{aligned}
F_{Z}^{(1)}\left(\phi^{---}, F_{i}^{++}\right) & =-F_{Z}^{(1)}\left(\phi^{\prime+}, F_{i}^{--}\right) \quad \text { and } \\
F_{Z}^{(1)}\left(\phi^{--}, F_{i}^{+}\right) & =-F_{Z}^{(1)}\left(\phi^{0}, F_{i}^{-}\right),
\end{aligned}
$$

whereas, for the 7 plet, the contribution from the following pairs are

$$
\begin{aligned}
F_{Z}^{(1)}\left(\phi^{----}, F_{i}^{+++}\right) & =-F_{Z}^{(1)}\left(\phi^{\prime \prime++}, F_{i}^{---}\right), \\
F_{Z}^{(1)}\left(\phi^{---}, F_{i}^{++}\right) & =-F_{Z}^{(1)}\left(\phi^{\prime+}, F_{i}^{--}\right) \quad \text { and } \\
F_{Z}^{(1)}\left(\phi^{--}, F_{i}^{+}\right) & =-F_{Z}^{(1)}\left(\phi^{0}, F_{i}^{-}\right) .
\end{aligned}
$$

For the singlet, there is only one contribution in $F_{Z}^{(1)}$ which is coming from the $\left(\phi^{-}, F_{i}^{0}\right)$ pair. Therefore, in all cases, the only nonzero contribution in $F_{Z}^{(1)}$ comes from the $\left(\phi^{-}, F_{i}^{0}\right)$ pair.

In addition, $F_{Z}^{(2)}$ in Eq. (26) is zero because the loop functions satisfy the relation,

$2 C_{24}\left(m_{S_{1}}^{2}, 0,0\right)+\frac{1}{2}=2 C_{24}\left(0, m_{S_{1}}^{2}, m_{S_{1}}^{2}\right)=B_{1}\left(0, m_{S_{1}}^{2}\right)$.

And Z couplings are $g_{Z \nu \bar{\nu}}=\frac{g}{2 \cos \theta_{W}}, g_{Z S_{1}}=-\frac{g \sin ^{2} \theta_{W}}{\cos \theta_{W}}$, and $g_{L}^{l}=\frac{g}{\cos \theta_{W}}\left(-\frac{1}{2}+\sin ^{2} \theta_{W}\right)$. Therefore, the total sum turns out to zero. 


\section{Box contribution}

The box contribution can be arranged into three parts,

$$
B=B^{(1)}+B^{(2)}+B^{(3)} .
$$

Here, $B^{(1)}$ is the contribution associated with a neutral fermion in the loop and involves a combination of one-loop box topologies Fig. 4 (left and center). It is given as

$$
\begin{aligned}
e^{2} B^{(1)}= & \frac{1}{16 \pi^{2}} \sum_{i, j=1}^{3}\left[\frac{\tilde{D}_{0}}{2} g_{e i}^{*} g_{i \mu} g_{e j}^{*} g_{j e}\right. \\
& \left.+D_{0} m_{F_{i}^{0}} m_{F_{j}^{0}} g_{e i}^{*} g_{e i}^{*} g_{j \mu} g_{j e}\right],
\end{aligned}
$$

where, $\tilde{D}_{0}=\tilde{D}_{0}\left(m_{F_{i}^{0}}, m_{F_{j}^{0}}, m_{\phi^{+}}, m_{\phi^{+}}\right)$and $D_{0}=D_{0}\left(m_{F_{i}^{0}}\right.$, $\left.m_{F_{j}^{0}}, m_{\phi^{+}}, m_{\phi^{+}}\right)$.

On the contrary, the charged fermions running in the loop give contribution to $B^{(2)}$ and are associated to Fig. 4 (left). It is given as

$$
\begin{aligned}
e^{2} B^{(2)}= & \frac{1}{32 \pi^{2}} \sum_{i, j=1}^{3} \sum_{F} \sum_{\phi_{1}, \phi_{2}} \\
& \times \tilde{D}_{0}\left(m_{F_{i}}, m_{F_{j}}, m_{\phi_{1}}, m_{\phi_{2}}\right) g_{e i}^{*} g_{i \mu} g_{e j}^{*} g_{j e} .
\end{aligned}
$$

The sum index $F$ ranges over the charged components of fermion multiplets, and $\phi_{1,2}$ indices range over the corresponding scalar components set by the $G$ Yukawa term in Eq. (2).

For the singlet, the only contribution is $B^{(1)}$ that comes from a box diagram that involves $\phi^{+}$and the neutral fermion $F_{i}^{0}$. For larger scalar and fermion multiplets, apart from the $B^{(1)}$ contribution also $B^{(2)}$ has contributions from charged fermions as follows. For the triplet, the box contribution $B^{(2)}$ involves $\phi_{1,2} \in\left\{\phi^{++}, \phi^{0}\right\}$ for $F=F_{i}^{+}$. For the 5 plet, $\phi_{1,2} \in\left\{\phi^{+++}, \phi^{\prime-}\right\}$ for $F=F_{i}^{++}$and $\phi_{1,2} \in$ $\left\{\phi^{++}, \phi^{0}\right\}$ for $F=F_{i}^{+}$. On the other hand, for the 7 plet, $\phi_{1,2} \in\left\{\phi^{++++}, \phi^{\prime \prime--}\right\}$ for $F=F_{i}^{+++}, \phi_{1,2} \in\left\{\phi^{+++}, \phi^{\prime-}\right\}$ for $F=F_{i}^{++}$, and $\phi_{1,2} \in\left\{\phi^{++}, \phi^{0}\right\}$ for $F=F_{i}^{+}$.

There is also a box contribution coming from the charged scalar $S_{1}^{+}$[Fig. 4 (right)], which is given as

$$
e^{2} B^{(3)}=-\frac{1}{32 \pi^{2} m_{S}^{2}} f_{e \tau}^{*} f_{\tau \mu} f_{e \tau}^{*} f_{\tau e} .
$$

\section{C. $\mu$ to $e$ conversion rate}

The conversion rate, normalized by the muon capture rate is [37-39]

$$
\begin{aligned}
& \mathrm{CR}(\mu-e, \text { nucleus }) \\
& =\frac{p_{e} E_{e} m_{\mu}^{3} G_{F}^{2} \alpha_{e m}^{3} Z_{\mathrm{eff}}^{4} F_{p}^{2}}{8 \pi^{2} Z \Gamma_{\text {capt }}} \\
& \quad \times\left\{\left|(Z+N)\left(g_{L V}^{(0)}+g_{L S}^{(0)}\right)+(Z-N)\left(g_{L V}^{(1)}+g_{L S}^{(1)}\right)\right|^{2}\right. \\
& \left.\quad+\left|(Z+N)\left(g_{R V}^{(0)}+g_{R S}^{(0)}\right)+(Z-N)\left(g_{R V}^{(1)}+g_{R S}^{(1)}\right)\right|^{2}\right\} .
\end{aligned}
$$

Here, $Z$ and $N$ are the number of protons and neutrons in the nucleus, $Z_{\text {eff }}$ is the effective atomic charge, $F_{p}$ is the nuclear matrix element, and $\Gamma_{\text {capt }}$ represents the total muon capture rate. $p_{e}$ and $E_{e}$ are the momentum and energy of the electron, which is taken as $\sim m_{\mu} \cdot g_{X K}^{(0)}$ and $g_{X K}^{(1)}(X=L, R$ and $K=V, S)$ in the above expression are given as

$$
\begin{aligned}
& g_{X K}^{(0)}=\frac{1}{2} \sum_{q=u, d, s}\left(g_{X K(q)} G_{K}^{(q, p)}+g_{X K(q)} G_{K}^{(q, n)}\right) \\
& g_{X K}^{(1)}=\frac{1}{2} \sum_{q=u, d, s}\left(g_{X K(q)} G_{K}^{(q, p)}-g_{X K(q)} G_{K}^{(q, n)}\right) .
\end{aligned}
$$

$g_{X K(q)}$ are the couplings in the effective Lagrangian describing the $\mu-e$ conversion,

$$
\begin{aligned}
\mathcal{L}_{\text {eff }}= & -\frac{G_{F}}{\sqrt{2}} \sum_{q}\left\{\left[g_{L S(q)} \bar{e}_{L} \mu_{R}+g_{R S(q)} \bar{e}_{R} \mu_{L}\right] \bar{q} q\right. \\
& \left.+\left[g_{L V(q)} \bar{e}_{L} \gamma^{\mu} \mu_{L}+g_{R V(q)} \bar{e}_{R} \gamma^{\mu} \mu_{R}\right] \bar{q} \gamma_{\mu} q\right\} .
\end{aligned}
$$

$G^{(q, p)}, G^{(q, n)}$ are the numerical factors that arise when quark matrix elements are replaced by the nucleon matrix elements,

$\left\langle p\left|\bar{q} \Gamma_{K} q\right| p\right\rangle=G_{K}^{(q, p)} \bar{p} \Gamma_{K} p, \quad\left\langle n\left|\bar{q} \Gamma_{K} q\right| n\right\rangle=G_{K}^{(q, n)} \bar{n} \Gamma_{K} n$

For the generalized KNT model, the $\mu-e$ conversion rate receives the $\gamma$ and $Z$ penguin contributions where the quark line is attached to photon and Z-boson lines in the respective penguin diagrams. It also does not receive any box contribution because there is no coupling between $\boldsymbol{\Phi}$
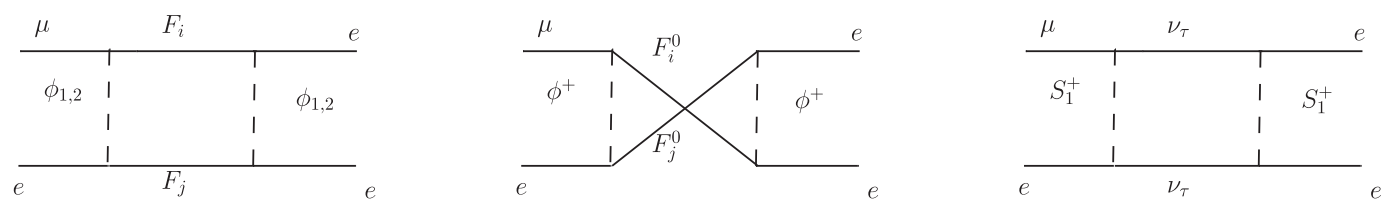

FIG. 4. One-loop box topologies associated to Feynman diagrams contributing to the $\mu \rightarrow e e \bar{e}$ process. 
and quarks. The relevant effective coupling for the conversion in this model is

$$
\begin{aligned}
& g_{L V(q)}=g_{L V(q)}^{\gamma}+g_{L V(q)}^{Z} \\
& g_{R V(q)}=\left.g_{L V(q)}\right|_{L \leftrightarrow R} \\
& g_{L S(q)} \approx 0, \quad g_{R S(q)} \approx 0 .
\end{aligned}
$$

The relevant couplings are

$$
\begin{aligned}
& g_{R V(q)}^{\gamma}=\frac{\sqrt{2}}{G_{F}} e^{2} Q_{q}\left[\left(A_{N D}^{(1)}+A_{N D}^{(2)}\right)-\left(A_{D}^{(1)}+A_{D}^{(2)}\right)\right], \\
& g_{L V(q)}^{\gamma}=\frac{\sqrt{2}}{G_{F}} e^{2} Q_{q}\left(A_{N D}^{(3)}-A_{D}^{(3)}\right) \\
& g_{R V(q)}^{Z}=-\frac{\sqrt{2}}{G_{F}} \frac{g_{L}^{q}+g_{R}^{q}}{2} \frac{F_{Z}^{(1)}}{m_{Z}^{2}}, \\
& g_{L V(q)}^{Z}=-\frac{\sqrt{2}}{G_{F}} \frac{g_{L}^{q}+g_{R}^{q}}{2} \frac{F_{Z}^{(2)}}{m_{Z}^{2}} .
\end{aligned}
$$

Here, $Q_{q}$ is the electric charge of the quarks and $\mathrm{Z}$ boson couplings to the quarks are

$$
\begin{aligned}
& g_{L}^{q}=\frac{g}{\cos \theta_{W}}\left(T_{3}^{q}-Q_{q} \sin ^{2} \theta_{W}\right), \\
& g_{R}^{q}=-\frac{g}{\cos \theta_{W}} Q_{q} \sin ^{2} \theta_{W} .
\end{aligned}
$$

Also, the relevant numerical factors for nucleon matrix elements are

$$
G_{V}^{(u, p)}=G_{V}^{(d, n)}=2, \quad G_{V}^{(d, p)}=G_{V}^{(u, n)}=1 .
$$

In the near degenerate limit, there will be a cancellation in the $A_{D}, A_{N D}$, and $F_{Z}$ contributions for triplet, 5-plet, and 7-plet cases as pointed out in Secs. III A, III B 1, and III B 2 . Therefore, the $\mu-e$ conversion rate will be also suppressed compared to the $\mu \rightarrow e e \bar{e}$ process in the KNT model. In addition, one can also consider novel charged LFV conversion rates suggested in $[40,41]$.

\section{RESULT AND DISCUSSION}

\section{A. Viable parameter space}

The parameter space of the generalized KNT model for a singlet, triplet, 5 plet, and 7 plet in the near degenerate limit is taken as $\left\{f_{\alpha \beta}, g_{i \alpha}, M_{F_{1,2,3}}, M_{\phi}, m_{S}, \lambda_{S}\right\}$ which enter into the neutrino mass generation in Eq. (6).

Here, we briefly present the collider constraints and future reach on the masses of the fermion and scalar multiplets in this model. The sensitivity study [42] on the process $e^{+} e^{-} \rightarrow S_{1}^{+} S_{1}^{-} \rightarrow l_{\alpha}^{+} l_{\beta}^{-}+E_{\text {miss }}$ in the KNT model at the future International Linear Collider (ILC) with $\sqrt{s}=1 \mathrm{TeV}$ showed that $m_{S} \gtrsim 240 \mathrm{GeV}$. On the other hand, it was shown in [43] that for the trilepton final states via $p p \rightarrow l^{ \pm} l^{ \pm} S_{1}^{-*} \rightarrow l^{ \pm} l^{ \pm} l^{ \pm}+E_{\text {miss }}$ at LHC with $\sqrt{s}=14 \mathrm{TeV}$ and luminosity, $L=300 \mathrm{fb}^{-1}$, the discovery reach for $S_{1}^{+}$increases up to $m_{S} \lesssim 4 \mathrm{TeV}$.

In addition, we have $F_{1}^{0}$ to be a DM candidate and that sets $M_{\phi}>M_{F_{1}}$. Based on searches of disappearing track signatures from long-lived charginos that are nearly mass degenerate with a neutralino at LHC with $\sqrt{s}=14 \mathrm{TeV}$ and $L=36.1 \mathrm{fb}^{-1}$ [44], we can reinterpret the exclusion limits for fermion components as $m_{F_{1}^{ \pm}} \gtrsim 600 \mathrm{GeV}$ for a lifetime, $\tau_{F_{1}^{ \pm}}=1 \mathrm{~ns}$. Moreover, for winolike minimal DM models that resemble fermion multiplets of the KNT model, a future collider with $\sqrt{s}=100 \mathrm{TeV}$ and $L=$ $3 \mathrm{ab}^{-1}$ [45] will improve this limit to $m_{F_{1}^{0}} \gtrsim 3.2 \mathrm{TeV}$. Besides, the multicharged component of the scalar multiplet, for example, $\phi^{++}$, can be produced via $q q^{\prime} \rightarrow$ $W^{+} \phi^{++} \phi^{-}$and consequently, will have the cascade decay, $\phi^{++} \rightarrow \phi^{+} W^{+*}$ etc., that will lead to multilepton final states and missing energy. The condition $M_{\phi}>M_{F_{1}}$ then also sets $M_{\phi} \gtrsim 3.2 \mathrm{TeV}$.

We scan over $M_{F_{1}} \in(1,50) \mathrm{TeV}, \quad M_{F_{2.3}} \in M_{F_{1}}+$ $(1,10) \mathrm{TeV}, \quad M_{\phi} \in(10,100) \mathrm{TeV}, \quad m_{S} \in(500 \mathrm{GeV}$, $50 \mathrm{TeV})$, and $\lambda_{S} \in(0.001,0.1)$. The Yukawa couplings, $f_{\alpha \beta}$ and $g_{i \alpha}$, are chosen so that they satisfy the low energy neutrino constraints. Afterwards, the rate of charged LFV processes are determined for all cases in this near degenerate limit. Although the generalized KNT model can contain a viable DM candidate, here we have studied charged LFV aspects of the model. In the companion paper [46], we show that for a standard freeze-out scenario, the DM relic density constraint leads to a very small window of mass at the $\mathrm{TeV}$ range but if the DM content of the Universe is set by a nonthermal process, the constraint on the mass can be relaxed.

\section{B. Charged LFV processes}

We can see from Fig. 5 that the rate of $\mu \rightarrow e e \bar{e}$ is very large compared to the $\mu \rightarrow e \gamma$ rate and the $\mu-e$ conversion rate in $\mathrm{Au}$ and $\mathrm{Ti}$ nuclei. The main reason behind this suppressed rate in the $\mu \rightarrow e \gamma$ and $\mu-e$ conversion rate is the cancellations among several one-loop diagrams, as mentioned in Secs. III A and III B 1, which have rendered dipole $A_{D}^{(2)}$ and nondipole $A_{N D}^{(2)}$ contributions, associated with a photon line attached to charged fermions, into zero in the near degenerate limit. Moreover, there are also cancellations in $A_{D}^{(1)}$ and $A_{N D}^{(1)}$ in this limit as shown in Secs. III A and III B 1 . We have further elaborated these cancellation in subsequent paragraphs. Such a pattern of a larger $\mu \rightarrow e e \bar{e}$ rate compared to $\mu \rightarrow e \gamma$ and $\mu-e$ conversion rates was also pointed out in the context of different BSM models in [47-51], but, to the best of our knowledge, in the context of the KNT model with singlet, triplet, 5-plet and 7-plet cases, this pattern has not been previously noted. 

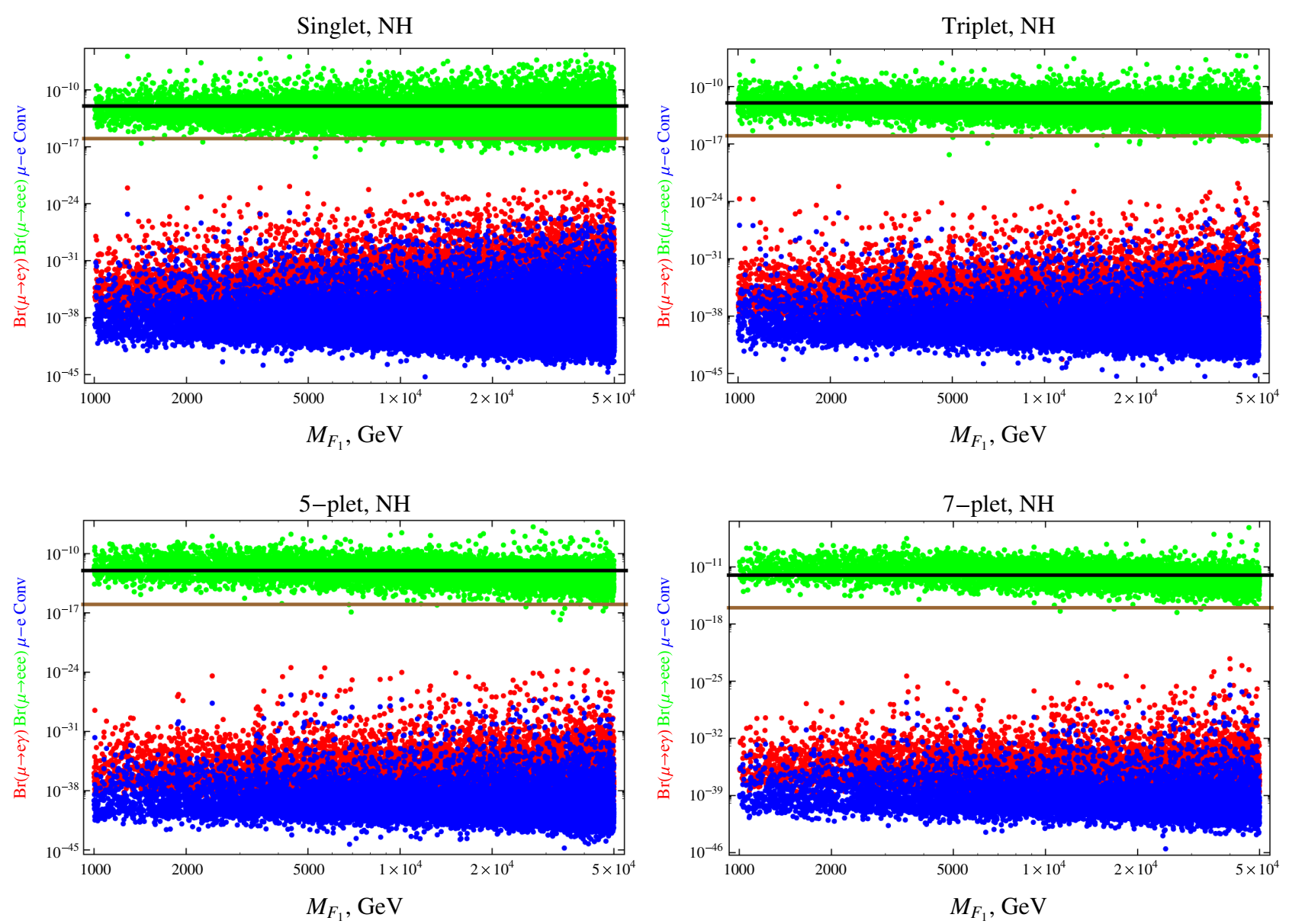

FIG. 5. LFV processes in singlet, triplet, 5-plet, and 7-plet cases for a normal hierarchy of neutrino masses. The graphs show the same pattern for an inverted hierarchy so they are not included here.

In addition, the contribution to the $Z$ penguin, $F_{Z}$ also receives several cancellations in one-loop diagrams, as mentioned in Sec. III B 2. On the other hand, such cancellations do not take place in the box contribution, $B$, and in the near degenerate limit, all box diagrams coherently add up for each of the singlet, triplet, 5-plet, and 7-plet cases. This can be seen from Fig. 6. Consequently, $A_{D}, A_{N D}$, and $F_{Z}$ enter into $\mu \rightarrow e \gamma, \mu \rightarrow e e \bar{e}$, and $\mu-e$ conversion rates, whereas $B$ also contributes into the $\mu \rightarrow$ $e e \bar{e}$ rate. Finally, we can see from Fig. 5 that for $M_{F_{1}}=$ 1-50 TeV range, part of the viable parameter space of the generalized KNT model is already excluded by the $\mu \rightarrow$ $e e \bar{e}$ rate set by SINDRUM, and the future Mu3e experiment will exclude almost all of the parameter space for all cases within this mass range. This implies that the masses of the BSM fermion and scalar particles of the KNT model had to be pushed beyond $50 \mathrm{TeV}$.

In the limit where the mass differences among the component fields in both scalar and fermion multiplets are negligible, there are cancellations among one-loop diagrams where the photon line is attached to the charged fermion component fields which is the consequence of $\sum_{i} q_{F_{i}}=0$ as the fermion multiplet is real. Therefore, the sum over all one-loop diagrams with a photon line attached with charged fermions, $A_{D}^{(2)}$ and $A_{N D}^{(2)}$, will be almost zero in triplet, 5-plet, and 7-plet cases. Note that, the singlet case does not have such a contribution because there is no charged fermion in its BSM particle content.

Likewise, there are cancellations among one-loop diagrams where the photon line is attached to charged scalars in the near degenerate limit for the triplet, 5 plet, and 7 plet. For singlet, the only contribution in $A_{D}^{(1)}$ and $A_{N D}^{(1)}$ comes from a one-loop diagram with a $\left(\phi^{-}, F_{i}^{0}\right)$ pair. On the other hand, apart from having this diagram common in all cases, there are nonzero contributions coming from $\left(\phi^{--}, F_{i}^{+}\right)$ and $\left(\phi^{-}, F_{i}^{0}\right)$ pairs for the triplet, $\left(\phi^{---}, F_{i}^{++}\right)$and $\left(\phi^{--}, F_{i}^{+}\right)$pairs for the 5 plet, and $\left(\phi^{----}, F_{i}^{+++}\right)$and $\left(\phi^{---}, F_{i}^{++}\right)$pairs for the 7 plet, respectively. So we can see that $A_{D}^{(1)}$ and $A_{N D}^{(1)}$ numerically do not deviate too much from each other in all cases.

As pointed out in Sec. III B 2, the total sum, $F_{Z}^{(1)}$, over Z-penguin diagrams containing charged fermions, is zero in the near degenerate limit. Therefore, in all cases, the nonzero Z-penguin contributions, $F_{Z}^{(2)}$ and $F_{Z}^{(3)}$, come from one-loop diagrams involving neutral fermions. 

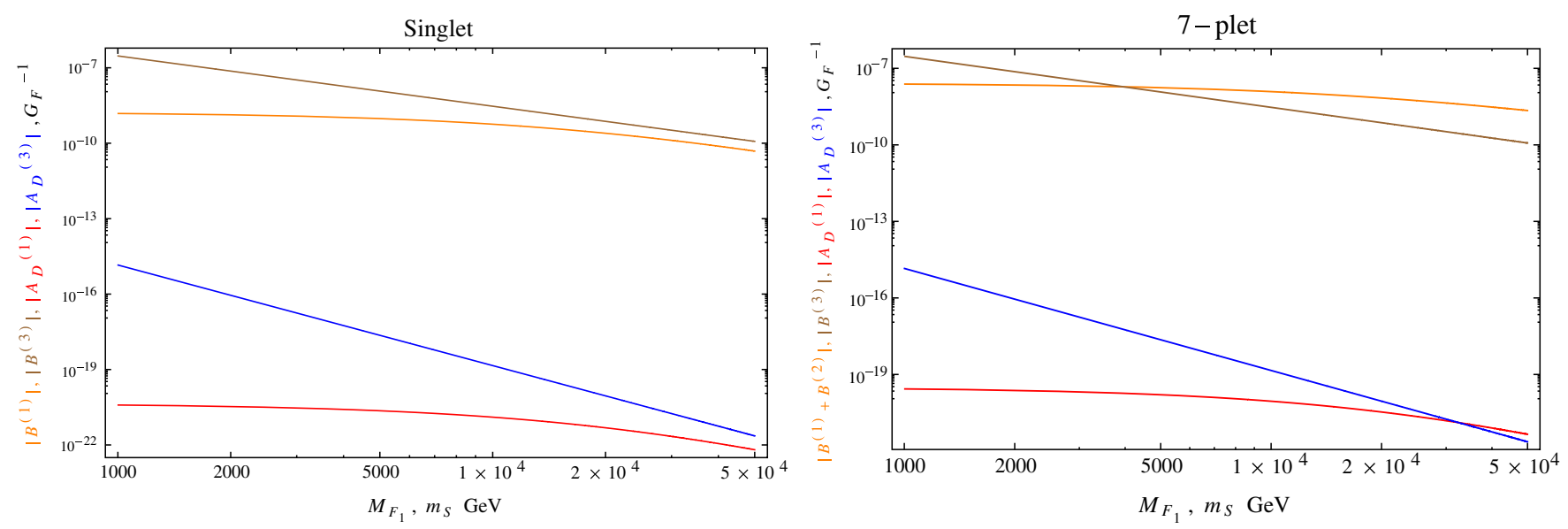

FIG. 6. (Left) Relative comparison among the dipole contributions, $A_{D}^{(1)}$ and $A_{D}^{(3)}$ and the box contributions, $B^{(1)}$ and $B^{(3)}$ in the $G_{F}^{-1}$ unit for the singlet case. Here we can see that box contributions are larger than dipole contributions. (Right) Similar comparison is made for the 7-plet case. As $A_{N D}$ behaves similarly as $A_{D}$ and also $F_{Z}$ is comparatively smaller than $A_{D}$ and $B$, we have not included them in the figure.

On the other hand, the box contribution, $B^{(1)}$ involves two diagrams containing neutral fermions in all cases. In contrast, instead of cancellation among one-loop box diagrams involving charged fermions, they all contribute to $B^{(2)}$. In the case of a triplet, 5 plet, and 7 plet, there are 4 , 8 , and 12 diagrams, respectively, that contribute coherently to $B^{(2)}$ in the near degenerate limit. Therefore, due to the cancellations among various one-loop diagrams, distinguishing different cases of a multiplet from a charged lepton flavor violation requires better precision.

Also in Fig. 6 (right), for the 7-plet case, the box contribution coming from diagrams with both neutral and charged fermions associated with the $G$ Yukawa sector, $\left|B^{(1)}+B^{(2)}\right|$, wins over $\left|B^{(3)}\right|$ associated with the $F$ Yukawa because, due to larger scalar and fermion multiplets, more particles enter into the loop, and therefore, $\left|B^{(1)}+B^{(2)}\right|$ becomes larger for $M_{F_{1}}$ than $B^{(3)}$ for $m_{S}$. A similar pattern can be seen also in dipole contributions.

In Fig. 5, which is basically the two-dimensional projection of a multidimensional parameter space, it can be seen that the rates of LFV processes increases for larger values of $M_{F_{1}}$, but this is due to the smaller value of $m_{S}$ for that $M_{F_{1}}$ value. Note that the decoupling behavior of a LFV process is apparent in Fig. 6, where we can see that, for example, the form factors for photonic dipole contribution $A_{D}$ and the box contribution decreases with increasing $M_{F_{1}}$ and $m_{S}$, which implies a decrease in the rate of the LFV processes at the large masses.

\section{CONCLUSION AND OUTLOOK}

We have investigated charged lepton flavor violating processes $\mu \rightarrow e \gamma, \mu \rightarrow e e \bar{e}$, and $\mu-e$ conversion in $\mathrm{Au}$ and Ti in the generalized KNT model with a singlet, triplet, 5 plet, and 7 plet. We have shown that due to the cancellation among several one-loop contributions to the photonic dipole term, photonic nondipole term, and Z-penguin term $A_{D}, A_{N D}$, and $F_{Z}$, respectively, the rates of $\mu \rightarrow e \gamma$ and $\mu-e$ conversion in $\mathrm{Au}$ and $\mathrm{Ti}$ become highly suppressed compared to $\mu \rightarrow e e \bar{e}$. This is due to the coherent addition of one-loop box diagrams where no cancellations take place and leads to a box contribution $B$ which enters into a $\mu \rightarrow e e \bar{e}$ process. As a consequence, we have seen that for the $M_{F_{1}}=1-50 \mathrm{TeV}$ mass range, the region of viable parameter space set by the neutrino sector is already excluded by the limit from SINDRUM, and the future Mu3e will have enough sensitivity to exclude almost all of the parameter space in this mass range and thus, push the mass of the lightest fermionic component larger than $50 \mathrm{TeV}$ in the generalized KNT model.

\section{APPENDIX: LOOP FUNCTIONS}

The loop functions relevant for the dipole and nondipole form factors from $\mu e \gamma$ vertex are

$$
\begin{aligned}
& F_{1}(x)=\frac{1-6 x+3 x^{2}+2 x^{3}-6 x^{2} \ln x}{6(1-x)^{4}} \\
& F_{2}(x)=\frac{2+3 x-6 x^{2}+x^{3}+6 x \ln x}{6(1-x)^{4}} \\
& G_{1}(x)=\frac{2-9 x+18 x^{2}-11 x^{3}+6 x^{3} \ln x}{6(1-x)^{4}} \\
& G_{2}(x)=\frac{16-45 x+36 x^{2}-7 x^{3}+6(2-3 x) \ln x}{6(1-x)^{4}} .
\end{aligned}
$$

In the following, we collect the Passarino-Veltman loop functions, 


$$
\begin{aligned}
& B_{1}\left(m_{1}, m_{2}\right)=-\frac{1}{2}-\frac{m_{1}^{4}-m_{2}^{4}+2 m_{1}^{4} \ln \frac{m_{2}^{2}}{m_{1}^{2}}}{4\left(m_{1}^{2}-m_{2}^{2}\right)^{2}}+\frac{1}{2} \ln \frac{m_{2}^{2}}{\mu^{2}} \\
& C_{0}\left(m_{1}, m_{2}, m_{3}\right)= \frac{m_{2}^{2}\left(m_{1}^{2}-m_{3}^{2}\right) \ln \frac{m_{2}^{2}}{m_{1}^{2}}-\left(m_{1}^{2}-m_{2}^{2}\right) m_{3}^{2} \ln \frac{m_{3}^{2}}{m_{1}^{2}}}{\left(m_{1}^{2}-m_{2}^{2}\right)\left(m_{1}^{2}-m_{3}^{2}\right)\left(m_{2}^{2}-m_{3}^{2}\right)} \\
& C_{24}\left(m_{1}, m_{2}, m_{3}\right)= \frac{\mathrm{A} 6)}{8\left(m_{1}^{2}-m_{2}^{2}\right)\left(m_{1}^{2}-m_{3}^{2}\right)\left(m_{2}^{2}-m_{3}^{2}\right)} \\
& \times\left[-2\left(m_{1}^{2}+m_{2}^{2}\right) m_{3}^{4} \ln \frac{m_{3}^{2}}{m_{1}^{2}}-\left(m_{3}^{2}-m_{1}^{2}\right)\right. \\
& \times\left(2 m_{2}^{4} \ln \frac{m_{2}^{2}}{m_{1}^{2}}+\left(m_{1}^{2}-m_{2}^{2}\right)\left(m_{2}^{2}-m_{3}^{2}\right)\right. \\
&\left.\left.\times\left(2 \ln \frac{m_{1}^{2}}{\mu^{2}}-3\right)\right)\right] \\
&(\mathrm{A} 7) \\
& \\
&
\end{aligned}
$$

$$
\begin{aligned}
\tilde{D}_{0}\left(m_{1}, m_{2}, m_{3}, m_{4}\right)= & \frac{m_{2}^{4} \ln \frac{m_{2}^{2}}{m_{1}^{2}}}{\left(m_{2}^{2}-m_{1}^{2}\right)\left(m_{2}^{2}-m_{3}^{2}\right)\left(m_{2}^{2}-m_{4}^{2}\right)} \\
& -\frac{m_{3}^{4} \ln \frac{m_{3}^{2}}{m_{1}^{2}}}{\left(m_{3}^{2}-m_{1}^{2}\right)\left(m_{3}^{2}-m_{2}^{2}\right)\left(m_{3}^{2}-m_{4}^{2}\right)} \\
& -\frac{m_{4}^{4} \ln \frac{m_{4}^{2}}{m_{1}^{2}}}{\left(m_{4}^{2}-m_{1}^{2}\right)\left(m_{4}^{2}-m_{2}^{2}\right)\left(m_{4}^{2}-m_{3}^{2}\right)}
\end{aligned}
$$

$$
\begin{aligned}
D_{0}\left(m_{1}, m_{2}, m_{3}, m_{4}\right)= & \frac{m_{2}^{2} \ln \frac{m_{2}^{2}}{m_{1}^{2}}}{\left(m_{2}^{2}-m_{1}^{2}\right)\left(m_{2}^{2}-m_{3}^{2}\right)\left(m_{2}^{2}-m_{4}^{2}\right)} \\
& -\frac{m_{3}^{2} \ln \frac{m_{3}^{2}}{m_{1}^{2}}}{\left(m_{3}^{2}-m_{1}^{2}\right)\left(m_{3}^{2}-m_{2}^{2}\right)\left(m_{3}^{2}-m_{4}^{2}\right)} \\
& -\frac{m_{4}^{2} \ln \frac{m_{4}^{2}}{m_{1}^{2}}}{\left(m_{4}^{2}-m_{1}^{2}\right)\left(m_{4}^{2}-m_{2}^{2}\right)\left(m_{4}^{2}-m_{3}^{2}\right)} .
\end{aligned}
$$

[1] T. P. Cheng and L. F. Li, Phys. Rev. Lett. 38, 381 (1977).

[2] T. P. Cheng and L. F. Li, Phys. Rev. D 16, 1425 (1977).

[3] S. T. Petcov, Yad. Fiz. 25, 641 (1977) [Sov. J. Nucl. Phys. 25, 340 (1977)]; Yad. Fiz. 25, 1336(E) (1977) [Sov. J. Nucl. Phys. 25, 698(E) (1977)].

[4] B. W. Lee and R. E. Shrock, Phys. Rev. D 16, 1444 (1977).

[5] W. J. Marciano and A. I. Sanda, Phys. Lett. 67B, 303 (1977).

[6] T. P. Cheng and L. F. Li, Phys. Rev. Lett. 45, 1908 (1980).

[7] C. S. Lim and T. Inami, Prog. Theor. Phys. 67, 1569 (1982).

[8] A. Blum and A. Merle, Phys. Rev. D 77, 076005 (2008).

[9] Y. Kuno and Y. Okada, Rev. Mod. Phys. 73, 151 (2001).

[10] S. Mihara, J. P. Miller, P. Paradisi, and G. Piredda, Annu. Rev. Nucl. Part. Sci. 63, 531 (2013).

[11] R. H. Bernstein and P. S. Cooper, Phys. Rep. 532, 27 (2013).

[12] A. Vicente, Adv. High Energy Phys. 2015, 686572 (2015).

[13] M. Lindner, M. Platscher, and F. S. Queiroz, Phys. Rep. 731, 1 (2018).

[14] L. Calibbi and G. Signorelli, Riv. Nuovo Cimento 41, 1 (2018).

[15] L. M. Krauss, S. Nasri, and M. Trodden, Phys. Rev. D 67, 085002 (2003).

[16] Y. Cai, J. Herrero-García, M. A. Schmidt, A. Vicente, and R. R. Volkas, Front. Phys. 5, 63 (2017).

[17] C. S. Chen, K. L. McDonald, and S. Nasri, Phys. Lett. B 734, 388 (2014).

[18] A. Ahriche, C.S. Chen, K. L. McDonald, and S. Nasri, Phys. Rev. D 90, 015024 (2014).
[19] A. Ahriche, K. L. McDonald, and S. Nasri, J. High Energy Phys. 10 (2014) 167.

[20] M. Gustafsson, T. Hambye, and T. Scarna, Phys. Lett. B 724, 288 (2013).

[21] A. Ahriche, K. L. McDonald, S. Nasri, and T. Toma, Phys. Lett. B 746, 430 (2015).

[22] A. M. Baldini et al. (MEG Collaboration), Eur. Phys. J. C 76, 434 (2016).

[23] U. Bellgardt et al. (SINDRUM Collaboration), Nucl. Phys. B299, 1 (1988).

[24] W. H. Bertl et al. (SINDRUM II Collaboration), Eur. Phys. J. C 47, 337 (2006).

[25] C. Dohmen et al. (SINDRUM II Collaboration), Phys. Lett. B 317, 631 (1993).

[26] P. W. Cattaneo (MEG II Collaboration), J. Instrum. 12, C06022 (2017).

[27] A. Bravar (Mu3e Collaboration), Nucl. Part. Phys. Proc. 287, 169 (2017).

[28] R. Donghia (Mu2e Collaboration), Nuovo Cimento Soc. Ital. Fis. 40, 176 (2017).

[29] M. Cirelli, N. Fornengo, and A. Strumia, Nucl. Phys. B753, 178 (2006).

[30] M. E. Peskin and T. Takeuchi, Phys. Rev. D 46, 381 (1992).

[31] R. Barbieri, A. Pomarol, R. Rattazzi, and A. Strumia, Nucl. Phys. B703, 127 (2004).

[32] C. Patrignani et al. (Particle Data Group), Chin. Phys. C 40, 100001 (2016). 
[33] J. Hisano, T. Moroi, K. Tobe, and M. Yamaguchi, Phys. Rev. D 53, 2442 (1996).

[34] E. Arganda and M. J. Herrero, Phys. Rev. D 73, 055003 (2006).

[35] A. Abada, M. E. Krauss, W. Porod, F. Staub, A. Vicente, and C. Weiland, J. High Energy Phys. 11 (2014) 048.

[36] E. Arganda and M. J. Herrero, arXiv:1403.6161.

[37] R. Kitano, M. Koike, and Y. Okada, Phys. Rev. D 66, 096002 (2002); 76, 059902(E) (2007).

[38] E. Arganda, M. J. Herrero, and A. M. Teixeira, J. High Energy Phys. 10 (2007) 104.

[39] A. Crivellin, M. Hoferichter, and M. Procura, Phys. Rev. D 89, 093024 (2014).

[40] W. Liao and X.H. Wu, Phys. Rev. D 93, 016011 (2016).

[41] T. Geib, A. Merle, and K. Zuber, Phys. Lett. B 764, 157 (2017).
[42] A. Ahriche, S. Nasri, and R. Soualah, Phys. Rev. D 89, 095010 (2014).

[43] D. Cherigui, C. Guella, A. Ahriche, and S. Nasri, Phys. Lett. B 762, 225 (2016).

[44] M. Aaboud et al. (ATLAS Collaboration), arXiv:1712.02118.

[45] M. Cirelli, F. Sala, and M. Taoso, J. High Energy Phys. 10 (2014) 033; 01 (2015) 41.

[46] T. A. Chowdhury and S. Nasri, arXiv:1802.05971.

[47] W. Grimus and L. Lavoura, Phys. Rev. D 66, 014016 (2002).

[48] E. H. Aeikens, W. Grimus, and L. Lavoura, Phys. Lett. B 768, 365 (2017).

[49] Y. Okada, K. i. Okumura, and Y. Shimizu, Phys. Rev. D 61, 094001 (2000).

[50] M. Aoki, S. Kanemura, T. Shindou, and K. Yagyu, J. High Energy Phys. 07 (2010) 084; 11 (2010) 49.

[51] M. Aoki, S. Kanemura, and K. Yagyu, Phys. Rev. D 83, 075016 (2011). 